\title{
EFETIVIDADE DA INTERVENÇÃO FONOAUDIOLÓGICA NO TEMPO DE ALTA HOSPITALAR DO RECÉM-NASCIDO PRÉ-TERMO
}

\section{Effectiveness of speech, language and hearing therapy in hospital discharge of pre-term newborns}

\author{
Cristiane Nogueira Costa ${ }^{(1)}$, Giovanna Ribeiro Serejo Lima ${ }^{(2)}$, Rafaelle Mousinho Jorge ${ }^{(3)}$, \\ Rosicler Aparecida Costa Guimarães Malta ${ }^{(4)}$, Kátia Nemr ${ }^{(5)}$
}

\begin{abstract}
RESUMO
Objetivo: verificar a efetividade da intervenção fonoaudiológica na diminuição do tempo de alta hospitalar do recém nascido pré-termo. Métodos: coleta de dados em 96 prontuários de recém-nascidos hospitalizados no Berçário Intermediário (BI) da Maternidade Marly Sarney no ano de 2000 a 2002 quando não havia atendimento fonoaudiológico e no ano de 2002 a 2004 quando ocorreu a implantação dos serviços fonoaudiológicos. Resultados: os recém-nascidos que tiveram intervenção fonoaudiológica tiveram o tempo de internação mais curto do que os que não receberam. Os que receberam: $88 \%$ de 1 a 10 dias, $8 \%$ de 11 a 20 dias, $2 \%$ de 41 a 50 dias e $2 \%$ de 61 a 70 dias. Os que não receberam: $47,9 \%$ de 1 a 10 dias, $17,7 \%$ de 11 a 20 dias, $10,4 \%$ de 21 a 30 dias, $9,4 \%$ de 31 a 40 dias, $9,4 \%$ de 41 a 50 dias, $3,1 \%$ de 51 a 60 dias e $2,1 \%$ de 61 a 70 dias. Esse aspecto foi estatisticamente significante ( $p$-valor < 0.000). Conclusão: os dados obtidos apontam para a efetividade da intervenção fonoaudiológica em recém-nascidos pré-termos. Houve associação entre menor tempo de internação hospitalar e presença de intervenção fonoaudiológica.
\end{abstract}

DESCRITORES: Recém-Nascido; Prematuro; Fonoterapia

\section{INTRODUÇÃO}

O termo prematuridade foi inicialmente conceituado por Yllpö em 1919. Eram considerados prematuros todos recém-nascidos ( $\mathrm{RN}$ ) vivos, nascidos com peso

(1) Fonoaudióloga do Centro Especializado de Fisioterapia e Fonoaudiologia, Fonoaudióloga da Associação Beneficente Cristã de São Luís; Especialização em Motricidade Orofacial.

(2) Fonoaudióloga do Sesiclínica de São Luís; Especialização em Motricidade Orofacial e em Audiologia Clínica.

(3) Fonoaudióloga do Hospital da Criança de São Luís, Fonoaudióloga da Clínica Integrada Médico Odontológica e Fonoaudióloga do Colégio Ninho das Águias; Especialização em Motricidade Orofacial.

(4) Fonoaudióloga do Centro de Atenção Integral a Saúde do Idoso, Fonoaudióloga da Associação Beneficente Cristã, Fonoaudióloga Prestadora de Serviço da Cervejaria Primus Schincariol e da Oleama (Oleaginosas do Maranhão); Especialização em Motricidade Orofacial e em Gerontologia; Pós-Graduada em Saúde da Família.

(5) Fonoaudióloga Professora da Universidade de São Paulo; Doutora em Psicologia Social pela Universidade de São Paulo. igual ou inferior a 2.500 gramas, conceito este que perdurou por um longo período. A prematuridade foi, e ainda é, um problema mundial, sua maior incidência se faz sentir nos países subdesenvolvidos ou em desenvolvimento, onde a inadequação e a falta de recursos promovem uma maior morbidade e conseqüente um alto índice de mortalidade, predominando a lei biológica, onde somente os mais fortes sobrevivem ${ }^{1}$.

Em 1970, a Academia Americana de Pediatria passou a definir como pré-termo todo RN vivo de gestação inferior a 38 semanas ou 266 dias, enquanto que a Organização Mundial de Saúde (OMS), com suporte das Escolas Pediátricas Européias conceitua como recém-nascido pré-termo (RNPT), todo RN vivo de gestação inferior a 37 semanas ou 259 dias, sempre a partir da data do parto, valor este expresso em semanas completas. Nos RNPT, o desenvolvimento que devia ocorrer ainda intra-útero ficou incompleto, e por este motivo estes bebês muitas vezes, irão precisar da ajuda de uma equipe de vários profissionais para adaptar-se ao seu novo meio: o externo ${ }^{1}$.

Contudo, a idade gestacional, como critério isolado, é um indicador de prontidão considerado pobre

Rev CEFAC, São Paulo, v.9, n.1, 72-8, jan-mar, 2007 
para sucção, porque há entre os bebês pré-termo uma considerável variabilidade, uma vez que alguns podem iniciar a alimentação oral com 32 semanas, enquanto outros só estarão prontos a partir da $36^{\underline{a}}$ semana. É necessário, então, antes de introduzir mamadeira ou sucção no seio, saber se o bebê pode coordenar o mecanismo deglutição/respiração, o que deve ser feito durante a alimentação por gavagem, inserindo-se uma chupeta própria ou dedo enluvado na sua boca, para estimular a sucção não-nutritiva; ao mesmo tempo, utiliza-se um monitor cardiorrespiratório para avaliar as respostas ao estímulo dado ${ }^{2}$.

Na década de 60, surgem as primeiras Unidades de Terapia Intensiva Neonatais (UTIN); com a inserção de tecnologia dentro das unidades, o ambiente começou a ficar cheio de equipamentos e com constante intervenção de pessoal médico e de enfermagem. Surge no final da década de 50 uma nova subespecialidade de Pediatria: a Neonatologia ${ }^{3}$.

Os índices de mortalidade dos recém-nascidos de alto risco vêm progressivamente diminuindo devido aos avanços tecnológicos das UTIN, dos aparelhos, da área medicamentosa e também, em um número cada vez maior aos profissionais especializados nesta área ${ }^{4}$.

Fonoaudiologia neonatal é uma área merecedora de destaque, que tende a se expandir cada vez mais e conquistar seu espaço na equipe multidisciplinar. Atualmente a atuação do fonoaudiólogo no berçário é, na maioria das vezes, desconhecida e pouco solicitada por parte dos profissionais ${ }^{5}$. Isso porque a inserção da fonoaudiologia nesse panorama é recente ${ }^{6}$.

A atuação fonoaudiológica em berçários normais, berçários de risco, e em UTIN, corresponde a um importante e novo campo da Fonoaudiologia, em que a atuação está voltada para os recém-nascidos (RN) normais (RN de termo - RNT) e de risco (RN prétermo - RNPT, baixo peso - RNBP, pequenos para a idade gestacional - RNPIG, RN portadores de patologias específicas com comprometimento do sistema sensório-motor-oral-SSMO) ${ }^{7}$.

Vários aspectos são considerados importantes no que diz respeito à atuação do fonoaudiólogo em berçário com RNT e têm despertado interesse em caracterizar as condições de alimentação do recémnascido ${ }^{5}$.

A nutrição corresponde a uma das maiores preocupações na assistência aos RNPT, sendo a sucção a maneira mais adequada e eficiente de oferecer os nutrientes necessários. Porém, estes RN têm uma imaturidade global, incluindo o sistema estomatognático, que dificulta a realização da função de sucção e alimentação por via oral ${ }^{8}$.

Existem dois tipos diferentes de sucção: a sucção normal, aquela realizada no seio materno para a obtenção de alimentos, e a sucção sem fins nutritivos, que são hábitos orais, tais como: sucções de chupeta, de lábios, dedos, bochechas ${ }^{9}$.
A sucção, seja ela nutritiva ou não, está diretamente ligada ao fator emocional. Por meio da sucção, a criança alimenta-se, interage com o meio e satisfaz-se emocionalmente. Quando a sucção é natural, isto é, realizado no seio materno, onde uma série de músculos interage e auxilia no desenvolvimento do sistema estomatognático ${ }^{9}$.

A atenção para os estados de consciência tem um significado importante para os profissionais que assistem ao bebê e seu desenvolvimento, que não apenas o de promover a melhora da sua saúde como principalmente, garantir a efetividade da estimulação ${ }^{10}$.

O aleitamento materno é sem dúvida, o melhor alimento para $\mathrm{RN}$ a termo saudável. O leite humano é altamente importante para atender também às necessidades do bebê pré-termo ou doente. Amamentar é a mais importante iniciativa que a mãe pode proporcionar ao seu filho. Amamentar é nutrir e é uma forte prova de amor ${ }^{11}$.

Em função de 0 aleitamento materno atender às necessidades fisiológicas nutricionais e psicossociais de todos os lactentes, o mesmo deve ser estimulado sempre que possível ${ }^{12}$.

No âmbito da Fonoaudiologia, a questão alimentar, vem requerendo bastante atenção deste profissional atuante em unidades neonatais, devido à impossibilidade dos $\mathrm{RNs}$ receberem o alimento por via oral ${ }^{13}$.

Algumas crianças nascem muito pequenas ou prematuras e podem enfrentar dificuldades na amamentação, por não estar presente o reflexo de sucção, não coordenarem bem as funções de sucção / respiração / deglutição, ou ainda não terem força para sugar ${ }^{14}$.

Em virtude da imaturidade fisiológica, esses recém-nascidos devem ser alimentados por sonda nasogástrica ou orogástrica. Existem práticas que facilitam a passagem da alimentação de gavagem (sonda oro ou nasogástrica) por via oral; uma das técnicas é a estimulação oral por meio de estímulos táteis e de sucção não-nutritiva (SNN). Essa estimulação deve ser baseada no estado nutricional e no ganho de peso do bebê, contribuindo sistematicamente para o desenvolvimento do $\mathrm{RN}^{15}$.

A longa permanência dos RNPT em hospitais apresenta efeitos deletérios que vão desde riscos para infecções até distúrbios afetivos ${ }^{10}$.

Pelo trabalho dos fonoaudiólogos, tem havido uma conscientização maior por parte dos pediatras, neonatologistas, neurologistas e enfermeiros, quanto a esse importante trabalho desenvolvido nos berçários e UTIN; contudo trata-se de um tema ainda bastante recente dentro da Fonoaudiologia no Brasil ${ }^{16}$.

A Fonoaudiologia incorpora-se como uma especialidade para a atenção integral ao bebê e à mãe, com o objetivo de promover os aspectos de aleitamento, alimentação e comunicação global, incluindo 
o vínculo mãe-bebê, com uma escuta diferenciada e aberta para as dificuldades e diferenças sociais ${ }^{17}$.

Atualmente, no Brasil, vem-se trabalhando com a visão de um novo paradigma, que é o de atenção humanizada à criança e à família, em função de avanços tecnológicos que têm aumentado à sobrevivência e as perspectivas de desenvolvimento de recém-nascidos prematuros. Novos procedimentos sistematizados estão sendo valorizados, influenciando a qualidade da interação mãe-bebê, a comunicação, audição, o desenvolvimento global e a adequação da alimentação ${ }^{18}$.

A atuação do fonoaudiólogo em berçário de alto risco é proporcionar ao recém-nascido uma alimentação segura, funcional e prazerosa, ou seja, na medida em que o recém-nascido fizer associação da sucção com a saciação e conseqüentemente com a coordenação de grupos sucção/deglutição/respiração, ele ganha peso e com isso favorece a alta hospitalar precoce e o seu desenvolvimento futuro ${ }^{6}$. Esses aspectos visam promover a capacidade do bebê se alimentar por via oral o mais precocemente possível e de modo mais seguro, observando o funcionamento global de seu organismo ${ }^{19}$.

Esta intervenção tem como objetivo central habilitar o bebê a se alimentar por via oral. Advindos deste, dois outros estão presentes e são interligados, objetivando melhorar as condições clínicas vigentes favorecendo a alta mais precoce e o desenvolvimento do futuro bebê ${ }^{10}$.

O objetivo dessa pesquisa foi verificar a eficácia da intervenção fonoaudiológica na diminuição do tempo de alta hospitalar do recém nascido pré-termo.

\section{MÉTODOS}

O presente trabalho constituiu-se de um estudo retrospectivo e foi realizado no Hospital e Maternida- de Marly Sarney, em São Luís do Maranhão.

O Hospital e Maternidade Marly Sarney é um dos dois hospitais que são maternidades reconhecidas pelo Ministério da Saúde e Unicef como hospitais amigos da criança, em São Luís. Realiza em média 7.000 partos por ano e possui em seu quadro funcional uma equipe multiprofissional.

Foram estudados os prontuários de 96 RNPT de ambos os gêneros, separados em dois grupos: aqueles ingressos no período de julho de 2000 a julho de 2002 quando os mesmos não recebiam Intervenção Fonoaudiológica, e os que possuíam prontuário de agosto de 2002 a agosto de 2004 quando começou a ser dado atendimento fonoaudiológico.

Como critérios de inclusão, foram incluídos todos os RN com idade gestacional inferior a 38 semanas, de ambos os gêneros. Nos critérios de exclusão, foram retirados do estudo aqueles bebês que apresentavam fenda palatina, lábio-leporino, fístula traqueoesofágica e bebês sindrômicos, com má-formação e com alterações neurológicas.

Os dados foram coletados em prontuários arquivados na Unidade Neonatal do referido Hospital e foram anotados em uma Ficha de Coleta de Dados previamente elaborada e testados em estudo piloto, onde foi levado em consideração o tempo de internação.

As variáveis coletadas foram: Idade Gestacional (IG); Peso ao nascer; Gênero; Tempo de alta do RN com e sem Intervenção Fonoaudiológica.

O presente estudo foi aprovado pelo Comitê de Ética em Pesquisa do Centro de Especialização em Fonoaudiologia Clínica sob o número 023/2005.

Os dados foram apresentados na forma de estatística descritiva com freqüência absoluta e relativa, e aplicação do teste estatístico Qui-quadrado, considerando-se como valor de significância valores menores ou iguais a $5 \%(p \leq 0.05)$.

Ficha de Coleta de Dados em Prontuário

Data da Coleta de Dados: I I

Número do Prontuário:

- Nome da Mãe:

- Idade Gestacional:

- Nome do Recém-Nascido:

- Data do Nascimento:

- Gênero: $\square$ Masculino ' I Feminino

- Peso ao Nascer:

- Peso no Momento da Internação:

- Tempo de Internação:

- Recebeu Estimulação Fonoaudiológica? $\square \operatorname{Sim} \square$ Não

- Em caso afirmativo:

- Início: I I__ Término: 1 


\section{RESULTADOS}

Os grupos foram constituídos com 46 RNPT que não receberam atendimento fonoaudiológico e 50 RNPT que receberam a intervenção. Quanto à idade gestacional de nascimento, os resultados obtidos foram: de 30 a 34 semanas 2,2\%, com 35 e 36 semanas $73,8 \%$ e 37 semanas $24,0 \%$, sem associação estatística com a presença ou não da intervenção fonoaudiológica (Tabela 1).

Quanto ao peso de nascimento dos recém-nascidos que receberam intervenção fonoaudiológica: nenhum apresentou-se com peso entre $500 \mathrm{~g} \mathrm{a}$ $1000 \mathrm{~g}, 90 \%$ de $1001 \mathrm{~g}$ a $2000 \mathrm{~g}, 10 \%$ de $2001 \mathrm{~g}$ a $3000 \mathrm{~g}$ (Tabela 2).

Já em relação ao peso de nascimento dos re- cém-nascidos que não receberam intervenção fonoaudiológica observou-se: 2,2\% com peso entre $500 \mathrm{~g}$ a $1000 \mathrm{~g}, 77,7 \%$ de $1001 \mathrm{~g}$ a $2000 \mathrm{~g}, 20 \%$ de $2001 \mathrm{~g}$ a $3000 \mathrm{~g}$ (Tabela 2 ).

Os recém-nascidos que tiveram intervenção fonoaudiológica obtiveram como peso após alta: $94 \%$ de $1600 \mathrm{~g}$ a $2600 \mathrm{~g}, 4 \%$ de $2601 \mathrm{~g}$ a $3600 \mathrm{~g}$ e $2 \%$ entre $3601 \mathrm{~g}$ a $4600 \mathrm{~g}$. Já os que não tiveram intervenção fonoaudiológica obtiveram como peso após a alta: $76 \%$ de $1600 \mathrm{~g}$ a $2600 \mathrm{~g}, 18 \%$ de $2601 \mathrm{~g}$ a $3600 \mathrm{~g}$ e $6 \%$ entre $3601 \mathrm{~g}$ a $4600 \mathrm{~g}$. (Tabela 3 ).

Em relação ao tempo de internação, observou-se que os bebês que receberam a intervenção fonoaudiológica, tiveram o tempo de internação mais curto em relação aos que não receberam, conforme a Tabela 4, com associação estatística ( $p$-valor <0.001).

Tabela 1 - Associação da amostra quanto à idade gestacional de nascimento e presença ou não de intervenção fonoaudiológica

\begin{tabular}{|c|c|c|c|c|c|c|}
\hline \multirow{3}{*}{ IDADE GESTACIONAL } & \multicolumn{4}{|c|}{ FONO } & & \\
\hline & \multicolumn{2}{|c|}{ SIM } & \multicolumn{2}{|c|}{ NÃO } & \multicolumn{2}{|c|}{ TOTAL } \\
\hline & $f$ & $\%$ & f & $\%$ & $F$ & $\%$ \\
\hline 30 semanas & 1 & 2,0 & - & - & 1 & 1,1 \\
\hline 34 semanas & - & - & 1 & 2,2 & 1 & 1,1 \\
\hline 35 semanas & 16 & 32,0 & 18 & 39,1 & 34 & 36,9 \\
\hline 36 semanas & 20 & 40,0 & 14 & 30,4 & 34 & 36,9 \\
\hline 37 semanas & 13 & 26,0 & 13 & 28,3 & 26 & 24,0 \\
\hline TOTAL & 50 & 54,3 & 46 & 45,7 & 96 & 100,0 \\
\hline
\end{tabular}

Tabela 2 - Associação da amostra quanto ao peso de nascimento dos recém-nascidos e presença ou não de intervenção fonoaudiológica

\begin{tabular}{|c|c|c|c|c|c|c|}
\hline \multirow{3}{*}{ PESO DE NASCIMENTO } & \multicolumn{4}{|c|}{ FONO } & & \\
\hline & \multicolumn{2}{|c|}{ SIM } & \multicolumn{2}{|c|}{ NÃO } & \multicolumn{2}{|c|}{ TOTAL } \\
\hline & $f$ & $\%$ & $f$ & $\%$ & $\mathbf{F}$ & $\%$ \\
\hline 500 a $1000 \mathrm{~g}$ & - & - & 1 & 2,2 & 1 & 1,1 \\
\hline 1001 a $2000 \mathrm{~g}$ & 45 & 90,0 & 36 & 78,3 & 81 & 84,4 \\
\hline 2001 a $3000 \mathrm{~g}$ & 5 & 10 & 9 & 19,5 & 14 & 14,5 \\
\hline TOTAL & 50 & 52,1 & 46 & 47,9 & 96 & 100,0 \\
\hline
\end{tabular}


Tabela 3 - Associação da amostra quanto ao peso após a alta hospitalar e presença ou não de intervenção fonoaudiológica

\begin{tabular}{cccccccc}
\hline & \multicolumn{3}{c}{ FONO } & \multicolumn{3}{c}{ TOTAL } \\
PESO APÓS A ALTA & \multicolumn{2}{c}{ SIM } & \multicolumn{2}{c}{ NÃO } & \multicolumn{2}{c}{ T } \\
& f & $\%$ & f & $\%$ & F & $\%$ \\
\hline 1600 a $2600 \mathrm{~g}$ & 47 & 94,0 & 34 & 76,0 & 81 & 84,5 \\
2601 a 3600 g & 2 & 4,0 & 9 & 18,0 & 11 & 11,5 \\
3601 a 4600 g & 1 & 2,0 & 3 & 6,0 & 4 & 4,0 \\
\hline TOTAL & 50 & 100,0 & 46 & 100,0 & 96 & 100,0 \\
\hline
\end{tabular}

Qui-quadrado $=7,39$

$\mathrm{p}<0,0248$ (significante)

$\mathrm{f}=$ freqüência absoluta

Tabela 4 - Associação da amostra quanto ao tempo de internação e presença ou não de intervenção fonoaudiológica

\begin{tabular}{|c|c|c|c|c|c|c|}
\hline \multirow{3}{*}{ TEMPO DE INTERNAÇÃO } & \multicolumn{4}{|c|}{ FONO } & & \\
\hline & \multicolumn{2}{|c|}{ SIM } & \multicolumn{2}{|c|}{ NÃO } & \multicolumn{2}{|c|}{ TOTAL } \\
\hline & $\mathbf{F}$ & $\%$ & f & $\%$ & f & $\%$ \\
\hline 1 a 10 dias & 44 & 88,0 & 2 & 4,3 & 46 & 47,9 \\
\hline 11 a 20 dias & 4 & 8,0 & 13 & 28,3 & 17 & 17,7 \\
\hline 21 a 30 dias & - & - & 10 & 21,7 & 10 & 10,4 \\
\hline 31 a 40 dias & - & - & 9 & 19,6 & 9 & 9,4 \\
\hline 41 a 50 dias & 1 & 2,0 & 8 & 17,4 & 9 & 9,4 \\
\hline 51 a 60 dias & - & - & 3 & 6,5 & 3 & 3,1 \\
\hline 61 a 70 dias & 1 & 2,0 & 1 & 2,2 & 2 & 2,1 \\
\hline TOTAL & 50 & 100,0 & 46 & 100,0 & 96 & 100,0 \\
\hline
\end{tabular}

\section{DISCUSSÃO}

Pelos dados obtidos nos prontuários pesquisados, a atuação fonoaudiológica teve início entre a 35ª e $36^{\mathrm{a}}$ semana de IG, dados estes que concordam com outros estudos realizados a cerca da atuação fonoaudiológica com RNPT ${ }^{1,2,7,8,10}$.

Vários autores sugerem que a atuação fonoaudiológica tenha início com a idade gestacional, quando seja possível iniciar a transição da alimentação gástrica para a alimentação por via oral, geralmente compreendida entre a $32^{\underline{a}}$ a 34를 semanas $^{1,2,7,10,15}$.

Em relação ao peso dos bebês, a literatura aponta uma média que varia entre 1400 a 1800 gramas, ao iniciar a estimulação da SNN. Associado ao peso, encontra-se ainda as idades gestacionais variando de 30a a 35" semanas, ocorrendo em todos os estudos pesquisados o peso apropriado para a IG $I^{1,2,7,8,10}$. Este dado não coincidiu com a presente pesquisa, haja vista, que o início da intervenção fonoaudiológica deu-se à medida que o bebê apresentou condições clínicas estáveis para tal, geralmente por volta de 1001 a 2000 gramas.

Alguns autores enfatizam como efeitos da SNN, o ganho de peso e a redução no período de transição para a alimentação oral ${ }^{20-23}$.

Os achados significantes do presente estudo quanto ao ganho de peso após intervenção fonoaudiológica concordam com os descritos na lite- 
ratura. Bebês estimulados com SNN obtêm maior ganho de peso e conseqüentemente alta hospitalar precoce ${ }^{1,2,4,6-8,10,11,15,16,19-25}$. Em estudos realizados com grupo controle, observou-se que os bebês estimulados com SNN levaram menor tempo na ingesta oral total de alimentação prescrita do que os bebês do grupo controle ${ }^{2}$.

Estudo recente, relata que o ganho de peso e a redução do período de transição para alimentação por via oral estão relacionados com os efeitos do trabaIho realizado com a SNN, o que deste modo, enfatiza a importância da atuação fonoaudiológica junto à equipe multidisciplinar ${ }^{7}$. A presente pesquisa aponta para esta direção, observada pela associação estatística significante entre menor tempo de internação e presença da intervenção fonoaudiológica.

Trata-se de uma área de atuação ainda recente e novas pesquisas devem ser desenvolvidas com o intuito de definir protocolos eficazes em relação à intervenção fonoaudiológica com neonatos prematuros.

\section{CONCLUSÃO}

Os dados obtidos apontam para a efetividade da intervenção fonoaudiológica com recém-nascido prétermo. Houve associação entre menor tempo de internação hospitalar e presença de intervenção fonoaudiológica.

\begin{abstract}
Purpose: to assess the efficacy of speech therapy in reducing hospital time in pre-term newborns. Methods: comparing 46 pre-term infants between 2000 and 2002 in Marly Sarney maternity when the speech therapy service was not available in that hospital for 50 pre-term infants between 2002 and 2004 when that service was initiated. Data was obtained from patient charts. Results: among the pre term newborns, those that received speech therapy had a shorter hospitalization time: $88 \%$ were discharged between day 1 and day $10,8 \%$ between day 11 and day 20 and just $2 \%$ stayed between 41 and 50 days and $2 \% 61$ and 70 days. Among those who did not undergo speech therapy just 47,9\% were discharged between day 1 and day $1017,7 \%$ of these patients were discharged between the $11^{\text {th }}$ and the $20^{\text {th }}$ day, $10,4 \%$ were discharged between the 21 st and 30 th days, $9,4 \%$ were discharged between 31 and 40 days, 9,4\% were discharged between 41 and 50 days, 3,1\% were discharged between 51 and 60 days and 2,1\% were discharged between 61 and 70 days. This aspect was statistically significant ( $p$-value $<0.000$ ). Conclusion: the data showed the efficacy of speech therapy with pre-term infants. There was an association between shorter hospitalization time and speech therapy presence.
\end{abstract}

KEYWORDS: Infant, Newborn; Infant, Premature; Speech Therapy

\section{REFERÊNCIAS}

1. Brock R. Recém-nascido prematuro, baixo de crescimento intra-uterino. In: Bassetto MCA, Brock R, Wajnsztejn R. Neonatologia: um convite à atuação fonoaudiológica. São Paulo: Lovise; 1998. p. 67-73.

2. Caetano LC, Fujinaga Cl, Scochi CGS. Sucção não nutritiva em bebês prematuros: estudo bibliográfico. Rev Latinoam Enfermagem. 2003; 11(2):232-6.

3. Almeida EC, Terra EMM, Holzer S, Benetti LAS, Gierwiatowski A, Gutierrez FS. Ficha neonatal: explicação detalhada das siglas, terminologia e patologias. In: Almeida EC, Modes LC, organizadores. Leitura do prontuário: avaliação e conduta fonoaudiológica com recém-nato de risco. Rio de Janeiro: Revinter; 2005. p. 1-21.

4. Ramos LB, Souza NB. Prontidão escolar em prétermo. Rev Fono Atual. 2001; 15:22-9.

5. Ribeiro A, Silva CCFAN, Salcedo PHT, Ferreira VJA. Crianças relação do APGAR em recém-nascido a termo com a eficiência da mamada. Rev Fonoaudiol
Bras. 2004; 4(1):1-3.

6. Delgado SE, Halpern R. Aleitamento materno de bebês pré-termo com menos de 1500 gramas: sentimentos e percepções maternos. Arq Méd. 2004; 7(2):5-28.

7. Neiva FCB. Sucção em recém-nascidos: algumas contribuições da fonoaudiologia. [periódico on line]. Disponível em:

URL: http://www.pediatriasaopaulo.usp.br/upload/ $\mathrm{html} / 482 /$ body $/ 10 . \mathrm{htm}$

8. Neiva FCB. Análise evolutiva do padrão de sucção e a influência da estimulação através da sucção não nutritiva em recém-nascido pré-termo [doutorado]. São Paulo (SP): Universidade de São Paulo; 2003.

9. Pereira ERBN, Trezza EMC. Identificação das atitudes dos pais e familiares frente ao uso da chupeta. J Bras Fonoaudiol. 2005; 5(23):381-6.

10. Hernandez AM. Atuação fonoaudiológica em neonatologia: uma proposta de intervenção. In: Andrade CRF, organizadora. Fonoaudiologia em ber- 
çário normal e de risco. v. 1. São Paulo: Lovise; 1996. p. 83-7.

11. Rios IJA. Técnicas de sucção nutritiva para recém-nascido prematuro. In: Rios IJA, organizadora. Conhecimentos essenciais para atender bem em fonoaudiologia hospitalar. São Paulo: Pulso; 2003. p. 43-98.

12. Fonseca RP, Ferreira VJA. Relação da pressão de sucção e da pega de bebês a termo com o aparecimento de fissuras mamilares no processo de amamentação natural. Rev CEFAC. 2004; 6(1):49-57. 13. Garzi RP, Cerruti VQ. Importância da adequada respiração no processo de alimentação do recémnascido pré-termo: relato de caso. Rev CEFAC. 2003; 5(1):63-7.

14. Gamburgo LJL, Munhoz SEM, Anstalden LG. Alimentação do recém-nascido: aleitamento natural, mamadeira e copinho. Rev Fono Atual. 2002; 20:39-47. 15. Salcedo PHT. Trabalho fonoaudiológico específico em berçário com estimulação sensório-motor-oral. In: Oliveira ST, organizadora. Fonoaudiologia hospitalar. São Paulo: Lovise; 2003. p. 123-36.

16. Monte CD. Atuação comprovada da fonoaudiologia em UTI neonatal. Anais - VIII Congresso Brasileiro de Fonoaudiologia; 2000 Out 04-07; Recife (PE): p. 165. 17. Delgado SE. A influência da cultura no aleitamento materno: reflexões para melhor promovê-la. Rev Fono Atual. 2002; 19:20-3.

18. Andrade ISN, Guedes ZCF. Sucção do recémnascido prematuro: comparação do método mãe-can- guru com os cuidados tradicionais. Rev Bras Saúde Matern Infant. 2005; 5(1):61-9.

19. Azevedo ACMPF. Intervenção fonoaudiológica na maternidade Balbina Mestrinho. [periódico on line]. Disponível em:

URL: http://www.niltonlins.br/extensao/downloads/ TOP_Projeto_Balbina_Mestrinho.doc.

20. Field T, Ignatoff E, Stringer S, Brennan J, Greenberg R, Widmayer S, Anderson GC. Nonnutritive sucking during tube feedings: effects on preterm neonates in an intensive care unit. Pediatrics. 1982; 70(3):381-4.

21. Case-Smith J. An efficacy study of occupational therapy with high-risk neonates. Am J Occup Ther. 1988; 42(8):499-506.

22. Sehgal SK, Prakash O, Gupta A, Mohan M, Anand NK. Evaluation of beneficial effects of nonnutritive sucking in preterm infants. Indian Pediatr. 1990; 27(3):263-6.

23. Mattes RD, Maone T, Wager-Page S, Beauchamp G, Bernbaum J, Stallings V, et al. Effects on sweet taste stimulation on growth and sucking in preterm infants. J Obstet Gynecol Neonatal Nurs. 1996; 25(5):407-14.

24. Lang S. Aleitamento do lactente: cuidados especiais. 1. ed. São Paulo: Santos; 1999. p. 13-5.

25. Silva RNM. Fatores que interferem na sucção/ deglutição/respiração do prematuro. In: Lopes SMB, Lopes JMA. Follow up do recém-nascido de alto risco. Rio de Janeiro: Medsi; 1999. p. 275-300.

RECEBIDO EM: 14/09/06

ACEITO EM: 09/01/07

Endereço para correspondência:

Rua do Passeio, 1004

São Luís - MA

CEP: $65015-370$

Tel: (98) 32214399 / 81358272

E-mail: rep.malta@elo.com.br

Rev CEFAC, São Paulo, v.9, n.1, 72-8, jan-mar, 2007 